\title{
LA CAPACIDAD DEL SECTOR INDUSTRIAL PARA GENERAR OCUPACIÓN
}

\author{
Sofía Méndez Villarreal * \\ El Colegio de México
}

\section{INTRODUCCIÓN}

LA IMPORTANCIA indudable que tiene la generación de empleos remunerados a medida que avanza el proceso de desarrollo ha dado lugar a diversos análisis del problema. En el presente ensayo no se pretende hacer una evaluación de las causas del problema del desempleo y el subempleo en los países subdesarrollados, como tampoco de las posibles medidas que se han sugerido para hacer frente a este problema, cuya magnitud es motivo de preocupación creciente en los países en desarrollo. El objetivo central del presente análisis es más modesto. Se intenta examinar las tendencias del nivel y estructura del empleo industrial a medida que avanza el proceso de industrialización.

El proceso de desarrollo económico implica transformación de la estructura productiva, así como también aumentos de los niveles de productividad. En consecuencia, consideraremos cada uno de estos aspectos e intentaremos posteriormente la cuantificación de los efectos que tanto los cambios estructurales como los cambios en la productividad tienen sobre el empleo industrial en el caso específico de México.

La importancia de conocer las tendencias del empleo industrial justifica el estudio de este problema, aun con la serie de limitaciones derivadas del método de análisis seguido y de la información estadística disponible. Sin embargo, vale la pena destacar que, a pesar de estas deficiencias y limitaciones, fue posible deducir algunas tendencias interesantes acerca del nivel y la estructura del empleo industrial.

\section{DESCRIPCIÓN DEL MÉTODO}

Como se menciona antes, uno de los objetivos finales es la determinación del volumen y la estructura de la ocupación que se genera en el sector industrial después de cierto período de tiempo. Para esto, se utilizan los siguientes supuestos:

a) La existencia de una asociación entre el nivel del ingreso per capita y la estructura del producto industrial. Se supone que los cambios estructurales del sector industrial se reflejarán en la distinta participación dentro del producto industrial de las industrias ligeras, intermedias

* Deseo expresar mi agradecimiento a Héctor Sosa Valderrama por sus sugerencias y comentarios acerca de la metodología seguida. 
y de capital, a los que denominaremos respectivamente grupos o estratos industriales de tipo $A, B$ y $C$. ${ }^{\text {. }}$

b) Que a medida que avanza el proceso de industrialización, habrá cambios en la productividad por hombre ocupado. Estos cambios en los niveles de productividad implican efectos divergentes sobre el volumen de ocupación. La magnitud de estos efectos dependerá del tipo de cambios estructurales y técnicos que tengan lugar.

A continuación se describen los pasos a seguir en la estimación de los efectos sobre el empleo industrial, derivados de cambios estructurales y técnicos.

\section{Cambios estructurales}

Se establece una determinada estructura de productividad, "típica" o promedio, correspondiente a cada uno de los grupos industriales $A$, $B$ y $C$, de tal modo que una vez determinados los posibles cambios de estructura se puedan deducir los niveles de empleo probables, con base en los niveles de productividad "típicos" de cada estrato industrial dadas ciertas proyecciones del producto industrial.

Con el fin de obtener lo que llamaremos la estructura "típica" de productividad por estratos industriales se considera una muestra de países (Brasil, México y el Reino Unido). La productividad se define como el cociente del valor agregado (a costo de factores) entre el número de personas ocupadas en cada estrato industrial. Dado que los coeficientes de productividad obtenidos están expresados en la unidad monetaria de cada país, se optó por transformar estos coeficientes en índices de productividad, tomando como base de comparación la productividad de las industrias de tipo $A$. A partir de los índices de productividad de cada país, se calculó el índice "típico", que es simplemente el promedio de los índices por países. Las estimaciones obtenidas se presentan en el cuadro 1.

Es interesante observar la diferencia en los niveles de productividad al considerar como denominador, solamente el número de obreros 0 , alternativamente, el de obreros y empleados. La diferencia es más notoria en el caso de las industrias productoras de bienes de capital (tipo $C$ ), debido probablemente a que estas industrias se caracterizan por emplear mayor proporción de empleados, personal administrativo, directivos, profesionistas, etc., los cuales no están conectados de manera directa con el proceso productivo. Para el presente análisis se considera exclusivamente la productividad de los obreros. Es evidente que si utilizáramos el índice de obreros y empleados, el volumen estimado de empleo generado sería mayor, ya que, en general, los índices de productividad de obreros y empleados son menores que los de productividad de obreros solamente (véase el cuadro 1 ).

De especial importancia en nuestro análisis es la estructura del índice de productividad "típico", en el que $A=100, B=163$ y $C=123$, lo que indica que las industrias de tipo $B$ tienen los mayores niveles de productividad. Por su parte, las industrias de capital (tipo $C$ ) muestran

${ }^{1} \mathrm{La}$ enumeración de las ramas industriales incluidas dentro de cada grupo industrial aparece en: Héctor Sosa V., Planificación del desarrollo industrial, México, Siglo XXI Editores, 1966, p. 22. 
Cuadro 1

Productividad POR GRUPOS DE RAMAS INDUSTRIALES

\begin{tabular}{|c|c|c|c|}
\hline & \multicolumn{3}{|c|}{ Grupo industriel } \\
\hline & $\overline{\mathbf{I}}$ & $\bar{B}$ & $\bar{c}$ \\
\hline \multicolumn{4}{|l|}{ Bragil } \\
\hline $\begin{array}{l}\text { Produotividad de ompleados y obroros } \\
\text { Cooficiente a/ } \\
\text { Indioe }\end{array}$ & $\begin{array}{l}164.9 \\
100\end{array}$ & $\begin{array}{l}208.4 \\
127\end{array}$ & $\begin{array}{l}196.5 \\
119\end{array}$ \\
\hline $\begin{array}{l}\text { Productividad de obreros } \\
\text { Cooficiente if } \\
\text { Indice }\end{array}$ & $\begin{array}{l}172.2 \\
100\end{array}$ & $\begin{array}{l}289.2 \\
168\end{array}$ & $\begin{array}{l}252.8 \\
147\end{array}$ \\
\hline \multicolumn{4}{|l|}{ K6xion } \\
\hline $\begin{array}{l}\text { Productividad de ompleados y obreros } \\
\text { Coeficiento b/ } \\
\text { Indioe }\end{array}$ & 100.2 & $\underset{164}{43.1}$ & $\begin{array}{l}19.4 \\
74\end{array}$ \\
\hline $\begin{array}{l}\text { Productividad de obreros } \\
\text { Coeficiente b/ } \\
\text { Indioe }\end{array}$ & 100 & 170.5 & ${ }_{103}^{32.5}$ \\
\hline \multicolumn{4}{|l|}{ Reino Unido } \\
\hline $\begin{array}{l}\text { Produotividad do empleados y obreroa } \\
\text { Condiciente of } \\
\text { Indioe }\end{array}$ & $\begin{array}{r}1449 \\
100\end{array}$ & $\begin{array}{r}1733 \\
120\end{array}$ & $\begin{array}{r}1281 \\
88\end{array}$ \\
\hline $\begin{array}{l}\text { Productividad de obrerob } \\
\text { Cooficiente of } \\
\text { Indioe }\end{array}$ & $\begin{array}{r}1493 \\
100\end{array}$ & $\begin{array}{ll}2 & 247 \\
& 151\end{array}$ & $\begin{array}{r}1792 \\
120\end{array}$ \\
\hline Productividad de los obrerog & 100 & 163 & 123 \\
\hline
\end{tabular}

Fuente: Naciones Unidas, Statistical Yearbook, Nueva York, 1966.

a Medido en miles de cruzeiros por persona ocupada.

b Medido en miles de pesos por persona ocupada.

c Medido en miles de libras esterlinas por persona ocupada.

d Indice promedio (típico).

menor productividad relativa con respecto a las de tipo $B$, aunque mayor que las de tipo $A$. Como se verá más adelante, esta situación tendrá efectos importantes en los resultados de empleo generado.

Para los fines de este análisis, no interesa dilucidar las razones de los diferentes niveles de productividad entre los estratos industriales $A, B$ y $C$, ya que el hecho de que el coeficiente sea menor en ciertas ramas, no refleja necesariamente funcionamiento ineficiente de las industrias, sino más bien características técnicas de los procesos industriales.

Dadas ciertas proyecciones del producto industrial y del ingreso per capita, es posible determinar la estructura industrial que estaría asociada al nivel de ingreso per capita proyectado. De tal modo que, conocida la estructura del producto industrial proyectado, se puede deducir el nivel y la estructura de la ocupación, dividiendo el valor de la producción industrial en cada uno de los estratos $A, B$ y $C$, entre los coeficientes de productividad "tipificados".

Una vez estimado el nivel de empleo que resulta de los cambios estructurales previstos, se procederá a compararlo con el que existiría, dadas las mismas proyecciones del producto industrial, pero sin considerar cambios de estructura. Esto permite cuantificar el efecto de los cambios estructurales sobre la ocupación. Estas estimaciones se presentan en los cuadros 3 y 4 . 


\section{Cambios técnicos}

Con el fin de poder cuantificar los efectos de los cambios estructurales, se supondrá que los cambios en la productividad son reflejo de cambios técnicos. Se considera que la productividad de cada estrato industrial tiene una tendencia "típica", deducible a partir de la observación de una muestra de países. Para estimar esta tendencia se utilizan datos sobre Chile, Colombia y México, como un indicador de la tendencia de la productividad en los países latinoamericanos.

La tendencia de la productividad puede deducirse comparando los niveles de productividad en los años extremos de un período elegido, digamos diez años. El coeficiente de productividad en el año base se obtiene dividiendo el valor agregado en cada estrato industrial entre el número de trabajadores correspondiente. Mientras que en el décimo año se ha obtenido multiplicando el valor agregado en el año base por el índice de producción física y dividiendo este resultado entre el número de trabajadores ocupados en ese mismo año.

Los resultados de los cálculos anteriores pueden observarse en el cuadro 2 , en el que se presentan también los porcientos de incremento de los coeficientes de productividad en cada uno de los países considerados. La tendencia "típica" o promedio de los porcientos de incremento obtenidos fue de: $50 \%$ en las industrias tipo $A ; 52 \%$ en las tipo $B ;$ y $34 \%$ en las tipo $C$.

Los incrementos de productividad estimados no deben interpretarse estrictamente como efecto de cambios tecnológicos reflejados en la productividad de la mano de obra. Siendo estos coeficientes de productividad el resultado de la relación entre la magnitud del cambio de la pro-

\section{Cuadro 2}

\section{CAMBIOS DE PRODUCTIVIDAD POR GRUPOS DE RAMAS INDUSTRIALES (CAMBIOS "TÉCNICOS")}

\begin{tabular}{|c|c|c|c|}
\hline & \multicolumn{3}{|c|}{ Grupo industrial } \\
\hline & A & B & c \\
\hline \multicolumn{4}{|l|}{ Chile } \\
\hline $\begin{array}{l}\text { Coeficiente de productividad } \\
1953 \\
1963 \\
\text { Poroiento de incremento }\end{array}$ & $\begin{array}{c}236.4 \\
350.1 \\
48\end{array}$ & $\begin{array}{c}329.6 \\
614.7 \\
86\end{array}$ & $\begin{array}{c}150.0 \\
182.5 \\
22\end{array}$ \\
\hline \multicolumn{4}{|l|}{ Colombia } \\
\hline $\begin{array}{l}\text { Coeficiente de productividad } \\
1953 \\
1963 \\
\text { Porciento do incremanto }\end{array}$ & $\begin{array}{l}8.62 \\
11.78 \\
37\end{array}$ & $\begin{array}{l}8.19 \\
10.98 \\
34\end{array}$ & $\begin{array}{l}5.42 \\
8.46 \\
55\end{array}$ \\
\hline \multicolumn{4}{|l|}{$\underline{N 6 \times i c 0}$} \\
\hline $\begin{array}{l}\text { Coeficiente de productividad } \\
1950 \\
1960 \\
\text { Porciento de incremento }\end{array}$ & $\begin{array}{l}12.39 \\
20.56 \\
66\end{array}$ & $\begin{array}{l}14.30 \\
19.47 \\
36\end{array}$ & $\begin{array}{l}12.40 \\
19.03 \\
53\end{array}$ \\
\hline Tendencia "tfpica" o promedio & 50 & 52 & 43 \\
\hline
\end{tabular}

Fuente: Naciones Unidas, Statistical Yearbook, Nueva York, 1966.

a Escudos de 1960 por trabajador ocupado.

b Miles de pesos colombianos de 1953 por trabajador ocupado.

c Miles de pesos mexicanos de 1950, por trabajador ocupado. 
ducción física, y del cambio en la ocupación, están influidos por factores tales como una mejor organización y administración, así como por factores institucionales y económicos que operan tanto del lado de la oferta como de la demanda. Sin embargo, para los fines del presente análisis, la tendencia considerada parece adecuada, puesto que lo que se persigue es determinar cómo varía, en promedio, la ocupación a medida que aumenta el producto industrial en términos reales.

Con el fin de estimar la variación en la ocupación derivada de lo que hemos denominado cambios técnicos, o sea cambios en los niveles de productividad, se procederá como en el caso de los cambios de estructura. Excepto que en este caso los coeficientes de productividad utilizados se ven aumentados en la proporción correspondiente a la tendencia "típica" de los cambios en la productividad. O sea, se dividirán los montos proyectados del producto industrial, que pueden considerarse con $o$ sin cambios de estructura, entre los coeficientes de productividad que se supone existirán diez años después.

Finalmente se procede a cuantificar el efecto conjunto de los cambios técnicos y de los cambios de estructura sobre el empleo industrial (cuadro 5). En esta alternativa se supone que la distribución del valor agregado industrial entre las industrias de tipo $A, B$ y $C$ es reflejo de los cambios estructurales dentro del sector industrial que corresponden al nivel de ingreso proyectado; en tanto que los coeficientes de productividad utilizados son los que, de acuerdo con la tendencia "típica", prevalecerán al final del período proyectado, o sea en diez años.

La cuantificación del efecto neto sobre el empleo derivado de los cambios técnicos y de estructura puede hacerse comparando los niveles de ocupación que prevalecerían suponiendo una misma tasa de crecimiento del ingreso, y suponiendo alternativamente que los cambios técnicos y de estructura tengan o no lugar. Estos resultados aparecen en el cuadro 6, en el cual se observa: a) el efecto neto de los cambios de estructura deducidos a partir de las diferencias entre los resultados de los cuadros 4 y $3 ; b)$ el efecto neto total de los cambios técnicos y de estructura (obtenidos a partir de los resultados de los cuadros 4 y 5, y c) el efecto de los cambios técnicos que se obtuvieron por la diferencia entre los efectos totales y los efectos de cambios puramente estructurales.

\section{Aplicación al caso de México}

La aplicación de la metodología descrita al caso específico de México permite básicamente: 1) proyectar el nivel y composición del empleo industrial en México, que de acuerdo con las tendencias "típicas" existirá después de diez años, y 2) cuantificar los efectos de los cambios estructurales y técnicos sobre el volumen y la composición del empleo industrial. Esto último se estima comparando dicho nivel de empleo con el que existiría en el caso de que no se hubieran dado los cambios técnicos y de estructura.

La estimación del producto industrial para 1975, considerando el ingreso per capita $\left(Y_{p c}\right)$ para 1965 y el valor de la producción industrial $(P I)$, para el mismo año, se hace suponiendo que el primero crece al $3 \%$ y la segunda al $7 \%$ anual, en promedio, respectivamente, durante el período 1965-1975: 


\begin{tabular}{|c|c|c|c|}
\hline Año & $\begin{array}{l}\text { PBI } \\
\text { (Millones de pesos }\end{array}$ & corriontes) & $\begin{array}{c}\mathbf{Y}_{\mathrm{pc}} \\
(\mathrm{d} 6 \mathrm{lares})\end{array}$ \\
\hline 1965 & $252028^{a /}$ & لـ & $47 \mathrm{~b} /$ \\
\hline 1975 & & $154446^{\circ}$ & $632^{d /}$ \\
\hline
\end{tabular}

a Fuente: Nacional Financiera, La economía mexicana en cifras, 1970, cuadro 2.5, p. 25.

b PBI/Población.

c $\mathrm{PI}_{75}=\mathrm{PI}_{65}(1.07)^{10}$

$\mathrm{d}_{\mathrm{pc}(75)}=\mathrm{Y}_{\mathrm{pc}(65)}(1.03)^{10}$

Una vez estimado el producto industrial para 1975, se puede establecer la distribución del mismo por tipo de industrias $A, B$ y $C$, que corespondería al nivel de ingreso per capita proyectado. La participación de cada grupo industrial de acuerdo con las tendencias normales del proceso de industrialización varió de 49,30 y $21 \%$, respectivamente en 1965 , a 38,30 y $32 \%$ para las mismas industrias en $1975 .^{2}$

Determinado el orden de magnitud aproximado de los cambios estructurales, comentaremos ahora los resultados obtenidos para el caso de México.

\section{Efecto de los cambios de estructura}

Como se observa en el cuadro 3 , el nivel total de empleo industrial se ve disminuido como resultado de los cambios de estructura. Sin embargo, es sumamente interesante observar los cambios en la distribución del empleo entre los grupos industriales que se han definido.

En el caso de las industrias de bienes de capital (grupo $C$ ) cabe notar su gran aumento dentro del empleo industrial generado tanto en términos absolutos como relativos. La comparación de los volúmenes de ocupación generados con o sin cambios de estructura se pueden hacer comparando los resultados de la última columna de los cuadros 3 y 4, respectivamente. La cuantificación de esta diferencia se presenta en el cuadro 6.

Cuadro 3

MÉxico: VARIACIón EN EL EMPLEO CONSIDERANDO CAMBIOS DE ESTRUCTURA, 1965-1975

\begin{tabular}{|c|c|c|c|c|c|c|c|c|}
\hline & \multicolumn{4}{|c|}{$\begin{array}{l}\text { Es tructura del } \\
\text { produc to industrial }\end{array}$} & \multicolumn{2}{|c|}{$\begin{array}{l}\text { Coeficiente de } \\
\text { productividad } \\
\text { tipificado }\end{array}$} & \multicolumn{2}{|c|}{ Ocupaois $x^{b}$} \\
\hline & $\begin{array}{r}1965 \\
\text { Valor }\end{array}$ & $\%$ & $\begin{array}{r}1975 \\
\text { Valox }\end{array}$ & $\%$ & 1965 & 1975 & 1965 & 1975 \\
\hline $\begin{array}{l}A \\
B \\
C\end{array}$ & $\begin{array}{ll}38415 \\
23520 \\
16464\end{array}$ & $\begin{array}{l}49 \\
30 \\
21\end{array}$ & $\begin{array}{ll}58 & 689 \\
46 & 334 \\
49 & 423\end{array}$ & $\begin{array}{l}38 \\
30 \\
32\end{array}$ & $\begin{array}{l}24.6 \\
41.7 \\
37.8\end{array}$ & $\begin{array}{l}24.6 \\
41.7 \\
37.8\end{array}$ & $\begin{array}{r}1563 \\
564 \\
435\end{array}$ & $\begin{array}{ll}2 & 386 \\
1 & 111 \\
1 & 307\end{array}$ \\
\hline Total & 78399 & 100 & $154 \quad 446$ & 100 & & & 2562 & 4804 \\
\hline
\end{tabular}

a Millones de pesos.

b Miles de personas.

2 Estos resultados se determinaron por extrapolación, con base en la estructura "normal" analizada por Héctor Sosa V., op. cit., p. 22. 
Cuadro 4

MÉXICO: VARIACIÓN EN EL EMPLEO SIN CONSIDERAR CAMBIOS DE ESTRUCTURA, 1965-1975

\begin{tabular}{|c|c|c|c|c|c|c|c|c|}
\hline & \multicolumn{4}{|c|}{$\begin{array}{l}\text { Datruotura dol } \\
\text { produc to indus trial }\end{array}$} & \multicolumn{2}{|c|}{$\begin{array}{l}\text { Cooficiente de } \\
\text { productividad } \\
\text { tipifioado }\end{array}$} & \multicolumn{2}{|c|}{ Ooupacion } \\
\hline & $\begin{array}{l}1965 / \\
\text { valor }\end{array}$ & $\%$ & $\begin{array}{c}1975 \\
\text { Valor }\end{array}$ & $\%$ & 1965 & 1975 & 1965 & 1975 \\
\hline $\begin{array}{l}A \\
B \\
C\end{array}$ & $\begin{array}{l}38415 \\
23520 \\
16464\end{array}$ & $\begin{array}{l}49 \\
30 \\
21\end{array}$ & $\begin{array}{ll}75 & 679 \\
46 & 334 \\
34 & 433\end{array}$ & $\begin{array}{l}49 \\
30 \\
21\end{array}$ & $\begin{array}{l}24.6 \\
41.7 \\
37.8\end{array}$ & $\begin{array}{l}24.6 \\
41.7 \\
37.8\end{array}$ & $\begin{array}{r}1563 \\
564 \\
435\end{array}$ & $\begin{array}{ll}3 & 076 \\
1 & 111 \\
911\end{array}$ \\
\hline Total & $78 \quad 399$ & 100 & 154446 & 100 & & & 2562 & 5098 \\
\hline
\end{tabular}

a Millones de pesos.

b Miles de personas.

Como se puede observar, el aumento en el empleó proporcionado por las industrias de bienes de capital se ve más que neutralizado por el desplazamiento de mano de obra en las industrias ligeras (grupo $A$ ). Las industrias intermedias no tienen ningún efecto en este caso debido a que no se ven afectadas por cambios en su participación dentro del producto industrial.

La implicación práctica de la tendencia prevista de desplazamiento y generación de empleo, es que no basta pensar que se trataría de una simple redistribución de la mano de obra a favor de las industrias de bienes de capital. Es importante prever los diferentes grados de calificación y especialización de la mano de obra que se requerirá a fin de que su incorporación al proceso industrial no sea un factor limitante en la productividad y eficacia de las empresas industriales.

De la consideración de los cambios de estructura se pueden sustraer las siguientes conclusiones:

a) Hay un desplazamiento de mano de obra en las industrias ligeras, lo cual puede interpretarse como causa generadora de desocupación, especialmente de los obreros con niveles más bajos de calificación.

b) Es de esperar un gran dinamismo de las industrias de bienes de capital, lo cual permitirá la generación de nuevas oportunidades de empleo de mano de obra utilizada directa e indirectamente en el proceso productivo. Nótese que nos hemos estado refiriendo a coeficientes de productividad exclusivamente de los obreros.

c) Hay generación de desocupación considerando el sector industrial en su totalidad. Aunque debe notarse que el desplazamiento de mano de obra como consecuencia de cambios estructurales es bastante inferior al desempleo generado por otras causas, tales como los aumentos "normales" de productividad (véase el cuadro 6).

\section{Efecto de los cambios estructurales y técnicos.}

Con el fin de dar una visión más aproximada de los cambios totales en la ocupación, es importante considerar la influencia de los cambios tecnológicos, los cuales se ha supuesto que se reflejarán en los índices de productividad de la mano de obra. 
Cuadro 5

OCUPACIÓN INDUSTRIAL EN 1975, CONSIDERANDO CAMBIOS DE ESTRUCTURA Y CAMBIOS TÉCNICOS

\begin{tabular}{|c|c|c|c|c|}
\hline \multirow{2}{*}{$\begin{array}{l}\text { Grupo } \\
\text { indus trial }\end{array}$} & \multicolumn{2}{|c|}{$\begin{array}{c}\text { Estructura del } \\
\text { produoto industrial } \\
1975 \\
\end{array}$} & \multirow[t]{2}{*}{$\begin{array}{l}\text { Coeficiente de productividad } \\
\text { tipificado para } 1975\end{array}$} & \multirow[t]{2}{*}{$\begin{array}{l}\text { Nímero de trabajedores } \\
\text { ocupados } 1975\end{array}$} \\
\hline & Valor & $\%$ & & \\
\hline \multirow[t]{2}{*}{$\begin{array}{l}A \\
B \\
C\end{array}$} & $\begin{array}{ll}58 & 689 \\
46 & 334 \\
49 & 423\end{array}$ & $\begin{array}{l}38 \\
30 \\
32\end{array}$ & $\begin{array}{l}36.9 \\
63.4 \\
54.0\end{array}$ & $\begin{array}{r}1591 \\
731 \\
915\end{array}$ \\
\hline & 154446 & & & 3237 \\
\hline
\end{tabular}

a Millones de pesos.

b Miles de trabajadores.

।

Se considera que la tendencia "tipificada" o "normal" de los niveles de productividad obtenida, de acuerdo con la metodología descrita en la sección anterior, es aplicable al caso de México en el período de diez años proyectado. En consecuencia, se pueden cuantificar los efectos sobre el nivel y la estructura de la ocupación industrial derivados de cambios técnicos y estructurales. (Los resultados de estos cálculos se presentan en el cuadro 5.) En la estimación de este cuadro se utiliza la estructura industrial que se supone existirá en 1975. Para cuantificar la influencia de los cambios técnicos sobre el empleo industrial, se utilizaron, como denominadores, los coeficientes de productividad ajustados por la tendencia "típica" de la productividad, en cada estrato industrial, obtenida en el cuadro 2. Los resultados totales aparecen en la última columna del cuadro 5, y representan el nivel y estructura del empleo industrial que de acuerdo con las tendencias "normales" del proceso de industrialización existirán probablemente en 1975.

Cuadro 6

VARIACIÓN NETA EN LOS NIVELES DE OCUPACIÓN (Miles de trabajadores)

\begin{tabular}{|c|c|c|c|}
\hline $\begin{array}{l}\text { Grupo } \\
\text { industrial }\end{array}$ & $\begin{array}{c}\text { Efacto de los oambioo } \\
\text { de es tructura }\end{array}$ & $\begin{array}{l}\text { Ef oo to de los oambion } \\
\text { técnieos }\end{array}$ & $\begin{array}{l}\text { Efecto conjunto do } \\
\text { los cambios tónicoa } \\
\text { y de estruetura }\end{array}$ \\
\hline $\begin{array}{l}\mathbf{A} \\
B \\
C\end{array}$ & $\begin{array}{r}-690 \\
0 \\
+\quad 396\end{array}$ & $\begin{array}{l}-795 \\
-380 \\
-392\end{array}$ & $\begin{array}{r}-1485 \\
-\quad 380 \\
+\quad 4\end{array}$ \\
\hline Total & -294 & -1567 & -1861 \\
\hline
\end{tabular}

La variación neta en los niveles de ocupación derivada de cambios técnicos y estructurales se presenta en el cuadro 6. Los resultados obtenidos son sumamente interesantes ya que, no obstante las deficiencias estadísticas y las limitaciones metodológicas, muestran la tendencia que parece caracterizar el empleo industrial, a medida que avanza el proceso de industrialización. Se observa que el empleo en las industrias $A$ disminuye en forma importante, ya que representa sólo el $52 \%$ del nivel que prevalecería de no haberse dado cambios técnicos y estructurales. 
El empleo en las industrias $B$ se ve disminuido, debido básicamente a la influencia de cambios técnicos. Por último, tal vez el hallazgo más interesante y significativo es que el nivel de empleo en las industrias $C$ es ligeramente mayor al que hubiera existido de no haberse registrado cambios técnicos y estructurales. Por desgracia, el aumento del volumen de empleo generado es superado, con mucho, por el volumen de mano de obra desplazado. (Véase el cuadro 6.)

\section{CONSIDERACIONES FINALES}

Los resultados obtenidos a través del análisis cuantitativo parecen tener una contrapartida en la realidad. Es evidente que la composición de la fuerza de trabajo en lo que se refiere a calidad, varía según el grado de industrialización de las economías. No es coincidencia que en los países desarrollados, la mayor proporción de la fuerza de trabajo se encuentra ocupada en las industrias productoras de bienes de capital y de consumo duradero. En el caso de Inglaterra, por ejemplo, del total de obreros existentes en 1963 (6017600), el 36\% se ocupaba en las industrias $A$; el $21 \%$ en el grupo $B$; y el $43 \%$ en el grupo $C$. Aún más significativo es el hecho de que dentro del total de obreros y empleados (7 898500 ), las proporciones de ocupación fueron: $27 \%$ en las industrias $A ; 23 \%$ en las $B ;$ y $50 \%$ en las $C .^{3}$

Estas consideraciones, junto con el análisis llevado a cabo antes, dan base para pensar que las industrias productoras de bienes de capital, incluidas dentro del grupo $C$, se caracterizan por generar un volumen relativamente mayor de empleos directos y especialmente indirectos.

Asimismo, la utilización de mayores proporciones de mano de obra especializada y con mayores niveles de calificación y preparación, se traducen, en general, en mejores condiciones de trabajo y de vida para los trabajadores. Este fenómeno se observa, en mayor o menor grado, en los países industrializados, dado que a medida que se avanza en el proceso de industrialización, operando en condiciones de relativa eficiencia, se desplaza una proporción considerable de mano de obra no calificada, pero al mismo tiempo se van generando en las industrias intermedias y pesadas, oportunidades de empleo de mano de obra con mayores niveles de calificación.

La capacidad de la industria para generar estas oportunidades dependerá del grado y el ritmo del desarrollo, el cual a su vez se reflejará en los cambios de la estructura industrial y en la magnitud de los cambios técnicos. Asimismo, tendrá una importancia fundamental el tipo de industrias que se instalen y la elección tecnológica que se adopte para cada tipo de industrias.

Hemos tratado de destacar algunas de las tendencias del proceso de industrialización y sus efectos sobre la ocupación industrial. Observamos que hay un desplazamiento de mano de obra (suponemos que con niveles inferiores de calificación), y una generación de nuevas oportunidades de empleo, muy posiblemente con requerimientos de mayor calificación. Análisis a niveles más desagregados permitirían sin duda precisar los 
requerimientos de mano de obra en las nuevas industrias. Sin embargo, el problema no puede reducirse a atender la capacitación de mano de obra requerida. Es de fundamental importancia garantizar que los cambios técnicos y estructurales sean absorbidos por la economía en forma eficiente. Para esto es indispensable que se combinen en la medida de lo posible los objetivos de generación de empleo, y de expansión de la producción en condiciones de eficiencia. 